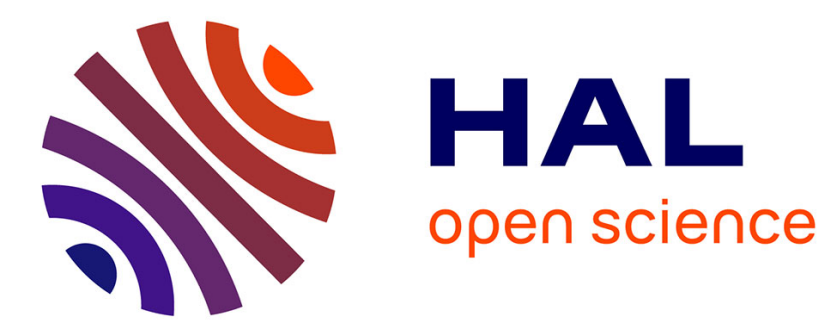

\title{
Multiple scattering approach to photoemission from the highest occupied molecular orbital of pentacene
}

\author{
N. Komiya, Keisuke Hatada, F. Ota, P. Krüger, T. Fujikawa, K. Niki
}

\section{To cite this version:}

N. Komiya, Keisuke Hatada, F. Ota, P. Krüger, T. Fujikawa, et al.. Multiple scattering approach to photoemission from the highest occupied molecular orbital of pentacene. Journal of Electron Spectroscopy and Related Phenomena, 2017, 220, pp.21-24. 10.1016/j.elspec.2017.04.002 . hal-01638858

\section{HAL Id: hal-01638858 \\ https://hal-univ-rennes1.archives-ouvertes.fr/hal-01638858}

Submitted on 7 Jun 2018

HAL is a multi-disciplinary open access archive for the deposit and dissemination of scientific research documents, whether they are published or not. The documents may come from teaching and research institutions in France or abroad, or from public or private research centers.
L'archive ouverte pluridisciplinaire HAL, est destinée au dépôt et à la diffusion de documents scientifiques de niveau recherche, publiés ou non, émanant des établissements d'enseignement et de recherche français ou étrangers, des laboratoires publics ou privés. 


\title{
Multiple scattering approach to photoemission from the highest occupied molecular orbital of pentacene
}

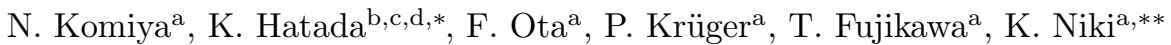 \\ ${ }^{a}$ Graduate school of Advanced Integration science, Chiba university, Chiba 263-8522, Japan \\ ${ }^{b}$ Département Matéiaux Nanosciences, Institut de physique de Rennes, UMR UR 1-CNRS 6251, Université de Rennes 1, 35042 Rennes \\ Cedex, France \\ ${ }^{c}$ Physics Division, School of Science and Technology, Università di Camerino, via Madonna delle Carceri 9, I-62032 Camerino (MC), Italy \\ ${ }^{d}$ INFN Laboratori Nazionali di Frascati, Via E Fermi 40, c.p. 13 , I-00044 Frascati, Italy
}

\begin{abstract}
Angle resolved photoemission spectroscopy (ARPES) gives detailed information about the surface electronic structures and oriented molecules. In the so-called orbital tomography method, the ARPES intensity is obtained by a simple Fourier transform of the initial state molecular orbital. While this method has given good results for a number of $\pi$-orbital systems, it is known to have strong limitations because the final state is approximated as a plane wave and thus photoelectron scattering is completely neglected. Here we propose an ARPES theory where the initial states are taken from a quantum chemistry method and the final states are calculated in multiple scattering theory. The initial molecular orbitals are reexpanded in a multi-site spherical wave basis and the multiple scattering potential is constructed from the ab initio charge density. These computations are performed algebraically using newly developed algorithms. The method is applied to pentance and the results are compared with the plane wave approximation.
\end{abstract}

Keywords: ARPES, Multiple scattering

2010 MSC: 00-01, 99-00

\section{Introduction}

In the last decades, intensive research has been done on organic semiconductors composed of $\pi$ conjugated organic molecules such as pentacence 1, 2. Compared to tradi-

5 tional inorganic materials, organic semiconductors are flexible, lightweight and cost-effective [3, 4]. As organic semiconductors absorb light in visible region and often show electronic conductivity, they have potential for the applications such as organic light emitting diodes (OLEDs), or-

10 ganic solar cells (OSCs), and organic field effect transistors (OFETs) [4, 5, 6].

Angle resolved photoelectron spectroscopy (ARPES) is a powerful tool to investigate the electronic structure of surfaces. For photon energies of a few tens of eV, the 15 photoelectron has a short inelastic mean free path and so the near surface region is probed.

Recently, P. Puschnig et al. 7] have developed the so- ${ }_{40}$ called orbital tomography method, by which the real space charge density of selected molecular orbitals can be reconstructed from the ARPES data. The orbital tomograph method relies on the plane wave (PW) approximation for the final state. Since the photoelectron energy is fairly low in ARPES, electron scattering at the molecular potential is expected to be strong and the validity of the PW approximation is questionable 8. One of the examples where the PW approximation fails is circular dichroism in ARPES intensity 9. Because the PW approximation gives exactly same intensity maps with a right and left handed light.

Photoelectron scattering effects can conveniently be taken account for using multiple scattering (MS) theory 10 11, 12. Usually single atom data is used for the construction of the scattering potentials. However, such potentials are inconsistent with the molecular orbital electronic structure. Here we overcome this shortcoming by constructing the MS potential from the electronic structure as given by a quantum chemistry method. For a gaussian-type orbital basis (as used, e.g. in the GAUSSIAN 09 code [13]). we have developed analytical formula and algorithms for mapping the molecular orbitals onto a MS spherical harmonics basis and have constructed the corresponding MS potential.

\footnotetext{
* Corresponding author

** Corresponding author

Email addresses: keisuke.hatada@univ-rennes1.fr (K.

Hatada), niki@chiba-u.jp (K. Niki)
} 


\section{Theory}

\subsection{The photoemission intensity}

From Fermi's golden rule, the photoemission intensity becomes

$$
I(\mathbf{k}) \propto \sum_{f}\left|\left\langle\psi_{f}|\Delta| \psi_{i}\right\rangle\right|^{2} \delta\left(\epsilon_{f}-\epsilon_{i}+\hbar \omega\right),
$$

where, $\psi_{i}$ is the initial state and $\psi_{f}$ is the final state. $\Delta$ is the electron-photon interaction operator and is expressed using the polarization vector $\hat{\epsilon}$, the momentum operator $\mathbf{p}$ and the position operator $\mathbf{r}$

$$
\Delta \propto \hat{\epsilon} \cdot \mathbf{p} \propto \hat{\epsilon} \cdot \mathbf{r} .
$$

In the PW approximation, the final state in Eq. (1) is a plane wave. Then, $I(\mathbf{k})$ simply becomes proportional to the Fourier transform of the initial state $\tilde{\phi}_{i}(\mathbf{k})$

$$
I(\mathbf{k}) \propto\left|\hat{\epsilon} \cdot \mathbf{k} \tilde{\phi}_{i}(\mathbf{k})\right|^{2} .
$$

If the energy of photoelectron is high enough, the scattering effects are not so strong and the PW approximation is justified to some extent. Also, scattering effects may be partly suppressed when the molecule is flat and the polarization vector is perpendicular to the molecular plane.

On the other hand, photoelectron scattering can be fully included in MS theory. Here we employ the usual ${ }^{65}$ muffin-tin (MT) approximation where the scattering potential is divided into spherically symmetric potential regions (MT spheres) located at atoms and the remaining "interstitial" regions where the potential is constant. The final state is a solution of the Lippmann-Schwinger equa- ${ }^{70}$ tion in this potential with appropriate boundary conditions. The photoemission intensity is written as 10

$$
\begin{aligned}
I(\mathbf{k}) \propto & \left|\left\langle\psi_{\mathbf{k}}^{-}|\Delta| \phi_{i}\right\rangle\right|^{2} \\
\approx & \mid \sum_{\alpha} e^{-i \mathbf{k} \cdot \mathbf{R}_{\alpha}} \sum_{A L_{1} L_{2} L_{3}} Y_{L_{1}}(\hat{\mathbf{k}}) \\
& \quad \times\left.\left[1+X+X^{2}+\cdots\right]_{L_{1} L_{2}}^{\alpha A} M_{L_{2} L_{3}}^{A}\right|^{2} .
\end{aligned}
$$

50 The superscript of $\psi_{\mathbf{k}}^{-}$indicates the imposed boundary condition, where the wave function is formed by fully interacting incoming waves and a plane wave which describes electron into the detector. $Y_{L}(\hat{\mathbf{k}})$ is a spherical harmonics, and $L \equiv(l, m)$. The matrix $X_{L L^{\prime}}^{\alpha \beta}$ describes the propagation of photoelectron from atom $\beta$ to atom $\alpha$ and scattering at atom $\alpha$, and $M_{L L^{\prime}}^{A}$ are the optical transition matrix elements of atom $A$.

\subsection{Algorithm for GAUSSIAN 09 output to MS calcula- tions}

\subsubsection{Spherical averages of charge density and potential}

We performed molecular orbital calculations by GAUSSIAN 09 to construct the scattering potential from the charge density and to obtain the initial state molecular orbital.

As basis sets are composed of Gaussian type orbitals (GTOs) in GAUSSIAN, the electron density can be expanded by products of GTOs. Any product of GTOs can be expanded by Hermite Gaussian functions which are defined by partial derivatives of a Gaussian function [14

$$
\Lambda_{t u v}\left(\mathbf{r}_{p}, p\right)=\frac{\partial^{t}}{\partial P_{x}^{t}} \frac{\partial^{u}}{\partial P_{y}^{u}} \frac{\partial^{v}}{\partial P_{z}^{v}} e^{-p r_{p}^{2}},
$$

where $t, u, v$ are orders of partial derivatives, $\mathbf{r}_{p}=\mathbf{r}-\mathbf{P}, \mathbf{P}$ is a point between two atomic sites, and the exponent $p$ is determined by the exponents of two GTOs. The spherical averages of Hermite Gaussian functions in terms of a given center $\mathbf{C}$ are obtained by integrating Eq. (5)

$$
\begin{aligned}
& \int \Lambda_{t u v}\left(\mathbf{r}_{p}, p\right) d \hat{\mathbf{r}}_{c}= \\
& \quad \frac{2 \pi}{p r_{c}} \sum_{t^{\prime} u^{\prime} v^{\prime}}{ }_{t} C_{t^{\prime} u} C_{u^{\prime} v} C_{v^{\prime}} F_{t-t^{\prime}, u-u^{\prime}, v-v^{\prime}} S_{t^{\prime} u^{\prime} v^{\prime}} .
\end{aligned}
$$

Here, we introduced the following two terms

$$
\begin{aligned}
& F_{t u v}=\frac{\partial^{t}}{\partial P_{x}^{t}} \frac{\partial^{u}}{\partial P_{y}^{u}} \frac{\partial^{v}}{\partial P_{z}^{v}} \frac{1}{R_{c p}} \\
& S_{t u v}=\frac{\partial^{t}}{\partial P_{x}^{t}} \frac{\partial^{u}}{\partial P_{y}^{u}} \frac{\partial^{v}}{\partial P_{z}^{v}} e^{-p R_{c p}^{2} \sinh \left(2 p r_{c} R_{c p}\right),}
\end{aligned}
$$

where $\mathbf{r}_{c}=\mathbf{r}-\mathbf{C}$ and $\mathbf{R}_{c p}=\mathbf{C}-\mathbf{P}$. To obtain the spherically averaged density, only a finite number of $F_{t u v}$ and $S_{\text {tuv }}$ term are needed. They can be calculated by using recurrence equations. Once the spherically averaged density is obtained, the spherically averaged scattering potential can be constructed using the local density approximation.

\subsubsection{Angular momentum expansion of an initial state}

In order to use an initial state molecular orbital in MS theory, we need its angular momentum expansion on the neighboring atomic sites. Any GTO has the following form

$$
\begin{aligned}
\chi(\mathbf{r}) & =x_{g}^{l} y_{g}^{m} z_{g}^{n} e^{-\alpha r_{g}^{2}} \\
& =e^{-\alpha\left(r_{c}^{2}+R_{c g}^{2}\right)} x_{g}^{l} y_{g}^{m} z_{g}^{n} e^{-2 \alpha \mathbf{r}_{c} \cdot \mathbf{R}_{c g}},
\end{aligned}
$$

where $l, m, n$ are integers which determine the angular dependence of a GTO, $\mathbf{r}_{g}=\mathbf{r}-\mathbf{G}, \mathbf{R}_{c g}=\mathbf{C}-\mathbf{G}, \mathbf{G}$ is the center of a GTO, and $\mathbf{C}$ is the center of the angular momentum expansion. Then, the polynomial and the later exponential in Eq.9. depend on the direction from the center $\mathbf{C}$. The polynomial can be expanded in spherical harmonics easily, and the exponential can also be expanded by using the analytic continuation of the Rayleigh expansion [15]

$$
\begin{aligned}
e^{-2 \alpha \mathbf{r}_{c} \cdot \mathbf{R}_{c g}}= & 4 \pi \sum_{L}(-1)^{l} \\
& \times i_{l}\left(2 \alpha r_{c} R_{c g}\right) Y_{L}\left(\hat{\mathbf{r}}_{c}\right) Y_{L}^{*}\left(\hat{\mathbf{R}}_{c g}\right),
\end{aligned}
$$

where $i_{l}$ is a modified spherical Bessel function. So, we need to express products of spherical harmonics by a series of spherical harmonics. 


\section{3. Results and discussion}

\subsection{Computational details}

We have applied the above theory to ARPES from the HOMO level of pentacene and compare the result with the PW approximation. A pentacene molecule consists of

five linearly fused benzene rings. The calculations are per-105 formed according to the experimental conditions [7. The photoelectron energy is $29.8 \mathrm{eV}$; the light is linearly ppolarized; the angle between the incident light $\mathbf{q}$ and the surface normal $\mathbf{n}$ is fixed to $\alpha=40^{\circ} ; \mathbf{q}, \mathbf{n}$ and photoemis-

${ }_{85}$ sion direction $\mathbf{k}$ lie in the same plane. The molecules lie $_{110}$ essentially flat on the surface. The long molecular axis is in the surface plane while the short axis is tilted by $\beta= \pm 25^{\circ}$ [7, see Fig 1. We use the ground state wave function of the neutral molecule for both the PW and the MS cal-

90 culations. For simplicity, scatterings off the substrate are ${ }_{115}$ neglected and ARPES intensity maps of a single molecule are computed.

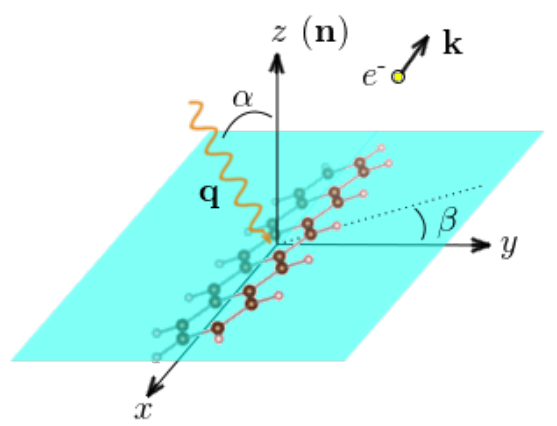

Figure 1: Schematic diagram of ARPES experiment.

\subsection{Calculated ARPES intensity maps and photoelectron angular distributions over whole azumithal angles}

The ARPES intensity maps calculated in the PW approximation and in the MS theory are shown in Fig. ${ }_{135}$ The result of the PW approximation (Fig, 2 ) agrees well with experiment (Fig 2 ) for this $\pi$-conjugated orbital and the chosen experimental conditions [7]. The MS result

(Fig 2 b) also agrees well with experiment, but shows a more complex pattern, indicating additional scattering effects.

For a detailed discussion of these effects, we have plotted the photoelectron angular distribution over whole azimuthal angles while fixing the polar angle at $25^{\circ}$ and $75^{\circ}$ in Fig 3 and 3 p respectively.

In Fig, 3 the average angle between the photoemission direction and the normal of molecular plane is fairly small. (Note that the molecular plane is tilted by $\pm 25^{\circ}$.) Then electron scattering is should be relatively weak. Compared to the PW approximation, the MS calculation shows a somewhat more complex angular distribution, but the overall shape is the same. On the other hand, in grazing emission (Fig $3 \mathrm{p}$ ), stronger scattering is expected. Indeed, the MS calculation gives additional peaks, and the overall shape is very different. We can therefore conclude that the importance of scattering effects considerably depends on the emission angle.

Multiple scattering theory fully accounts for the scatering of the photoelectron at the molecular potential, the neglect of which is a main source of error of the PW approximation. However, the present implementation of MS theory also has possible shortcomings because the MT approximation is used for the final state calculation. The HOMO of pentacene (Fig 4 ) is a $\pi$-orbital mainly consisting of carbon $2 p$ orbitals. For the calculation of the transition matrix elements $(M)$ the delocalized nature of such molecular orbitals is taken into account by expanding them into spherical waves centered on all sites. However, the carbon $2 p$ orbitals also extend into the so-called interstitial region, that is the space outside any atomic sphere. The contribution of this region is neglected in the calculation of the transition matrix elements. Second, the MT potential contains artificial jumps at the sphere boundaries which may cause spurious scattering effects. These points might be important for the present case of low en- (a) PW approximation

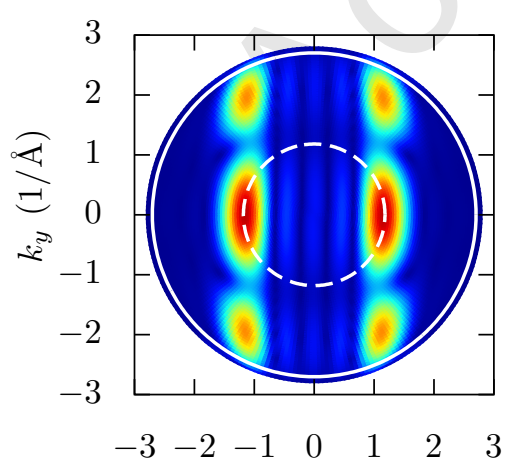

(b) MS theory

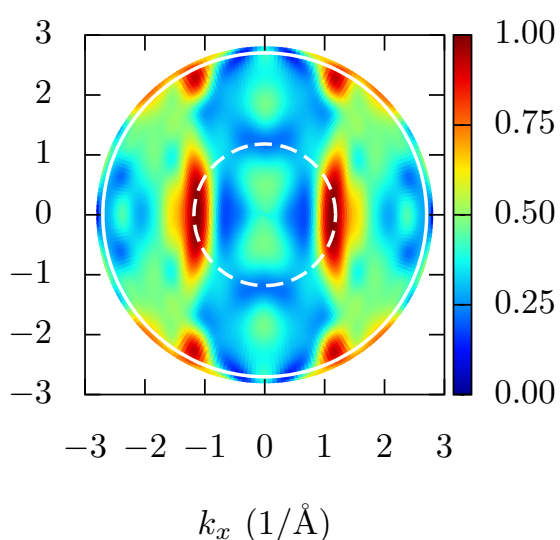

(c) Experiment

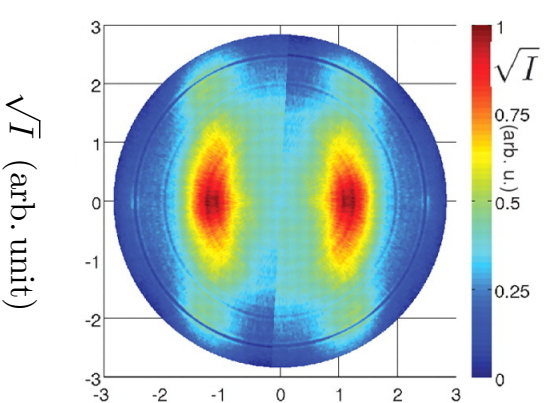

Figure 2: ARPES intensity map of pentacene. On the white solid and dashed line drawn in (a) and (b), the polar angles are fixed to $25^{\circ}$ and $75^{\circ}$ respectively. (a) PW calculation. (b) MS calculation. (c) Experimental data from Ref. [7], reprinted with permission. 
(a)

(b)

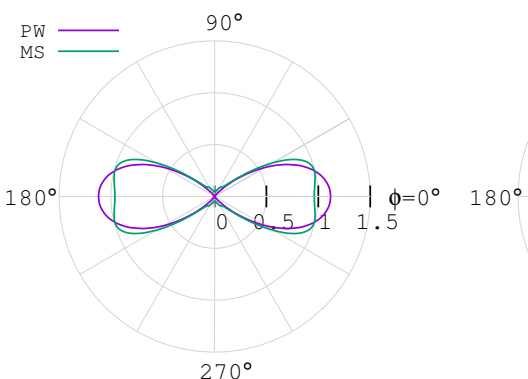

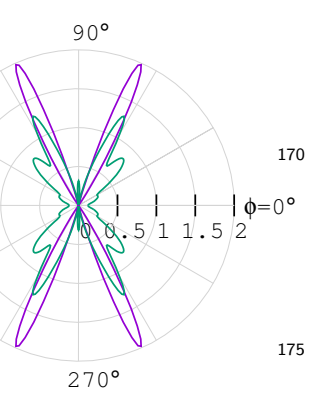

Figure 3: (a) Photoelectron angular distribution over whole azumithal angles. The green and red lines correspond to the $\mathrm{PW}_{180}$ and MS theory, respectively. The polar angle of emission direction is fixed at $25^{\circ}$, depicted with the white dashed line in Fig $2 \mathrm{a}$ and Fig 2 p. (b) Same as (a), for polar angle $75^{\circ}$, see the white solid line in Fig $2 \mathrm{a}$ and Fig $2 \mathrm{p}$.

ergy photoemission and should be corrected. This may be done by using the full-potential version of MS theory [16]. In full-potential MS theory, the MT spheres are replaced $_{190}$ space-filling atomic and empty cells. Thereby molecular potentials of any shape can be represented exactly.

As discussed before, some potential details are disregarded in the present calculation due to the MT approxi-195 mation. However, it should be kept in mind that the PW pproximation corresponds to the complete neglect of the molecular potential for the final state.

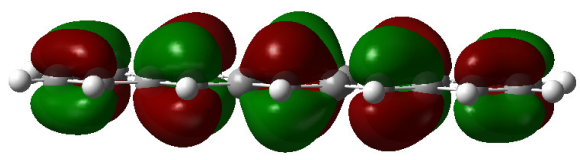

Figure 4: The HOMO of pentacene.

\section{Conclusion}

In summary we have proposed an improved multiple scattering theory for ARPES of oriented molecules. For this purpose, we have developed new algorithms for obtaining the spherically averaged scattering potential and the $\mathrm{e}^{215}$ angular momentum expansion of the initial state, which is taken from an ab initio molecular orbital calculation. We have tested the method on ARPES from the HOMO of pentacene and found good agreement with experiment. ${ }^{22}$ Compared with the PW approximation, the ARPES maps obtained in MS theory display additional features which we attribute to final state photoelectron scattering. However, in the present system, which is a favorable case for the validity of the PW approximation, the overall difference between PW and MS results is rather small. Due to finite experimental resolution, both calculations fit the experimental data equally well. Other systems and experimental geometries will be investigated in the future for a more clearcut comparison between the two approaches. In particular, large difference can be expected for molecules with appreciable emission perpendicular to the light polarization vector, because the $\mathrm{PW}$ approximation cannot account for this effect.

\section{References}

[1] S. K. T. Nishikawa, T. Takenobu, S. Mori, T. Shimoda, T. Mitani, H. Shimotani, N. Yoshimoto, S. Ogawa, Y. Iwasa, Nat. Mater. 3 (2004) 317.

[2] H. Kakuta, T. Hirahara, I. Matsuda, T. Nagao, S. Hasegawa, N. Ueno, K. Sakamoto, Phys. Rev. Lett. 98 (2006) 247601.

[3] Y. L. Loo, T. Someya, K. W. Baldwin, Z. Bao, P. Ho, A. Dodabalapur, H. E. Katz, J. A. Rogers, Proc. Natl. Acad. Sci. USA 99 (2002) 10252.

[4] S. R. Forrest, Nature 428 (2004) 911.

[5] K. Walzer, B. Maennig, M. Pfeiffer, K. Leo, Chem. Rev. 107 (2007) 1233.

[6] G. Holowitz, F. Garnier, A. Yassar, R. Hajlaoui, F. Kouki, Adv. Mater. 8 (1996) 52

[7] P. Puschnig, S. Berkebile, A. J. Fleming, G. Koller, K. Emtsev, T. Seyller, J. D. Riley, C. Ambrosch-Draxl, F. P. Netzer, M. G. Ramsey, Science 326 (2009) 702.

[8] A. M. Bradshaw, D. P. Woodruff, New J. Phys. 17 (2015) 013033.

[9] M. Dauth, M. Graus, I. Schelter, M. Wießner, A. Schöll, F. Reinert, S. Kümmel, Phys. Rev. Lett. 117 (2016) 183001

[10] H. Shinotsuka, H. Arai, T. Fujikawa, Phys. Rev. B 77 (2008) 085404.

[11] S. Nagamatsu, S. Kera, K. Okudaira, T. Fujikawa, N. Ueno, e-J. Surf. Sci. Nanotech. 3 (2005) 461.

[12] M. H. Shang, M. Nagaosa, S. Nagamatsu, S. K. Shunsuke Hosoumi, T. Fujikawa, N. Ueno, J. Electron Spectrosc. Relat. Phenom. 184 (2011) 261

[13] M. J. Frisch, G. W. Trucks, H. B. Schlegel, G. E. Scuseria, M. A. Robb, J. R. Cheeseman, G. Scalmani, V. Barone, B. Mennucci, G. A. Petersson, H. Nakatsuji, M. Caricato, X. Li, H. P. Hratchian, A. F. Izmaylov, J. Bloino, G. Zheng, J. L. Sonnenberg, M. Hada, M. Ehara, K. Toyota, R. Fukuda, J. Hasegawa, M. Ishida, T. Nakajima, Y. Honda, O. Kitao, H. Nakai, T. Vreven, J. A. Montgomery, Jr., J. E. Peralta, F. Ogliaro, M. Bearpark, J. J. Heyd, E. Brothers, K. N. Kudin, V. N. Staroverov, R. Kobayashi, J. Normand, K. Raghavachari, A. Rendell, J. C. Burant, S. S. Iyengar, J. Tomasi, M. Cossi, N. Rega, J. M. Millam, M. Klene, J. E. Knox, J. B. Cross, V. Bakken, C. Adamo, J. Jaramillo, R. Gomperts, R. E. Stratmann, O. Yazyev, A. J. Austin, R. Cammi, C. Pomelli, J. W. Ochterski, R. L. Martin, K. Morokuma, V. G. Zakrzewski, G. A. Voth, P. Salvador, J. J. Dannenberg, S. Dapprich, A. D. Daniels, Ö. Farkas, J. B. Foresman, J. V. Ortiz, J. Cioslowski, D. J. Fox, Gaussian 09 Revision E.01, gaussian Inc. Wallingford CT 2009.

[14] T. Helgaker, P. Jorgensen, J. Olsen, Molecular ElectronicStructure Theory, New York: John Wiley and Sons Inc., 2000.

[15] M. Stone, P. Goldbart, Mathematics for Physics: A Guided Tour for Graduate Students, CAMBRIDGE, 2009.

16] K. Hatada, K. Hayakawa, M. Benfatto, C. R. Natoli, J. Phys. Condens. Matter, 22 (2010) 185501. 